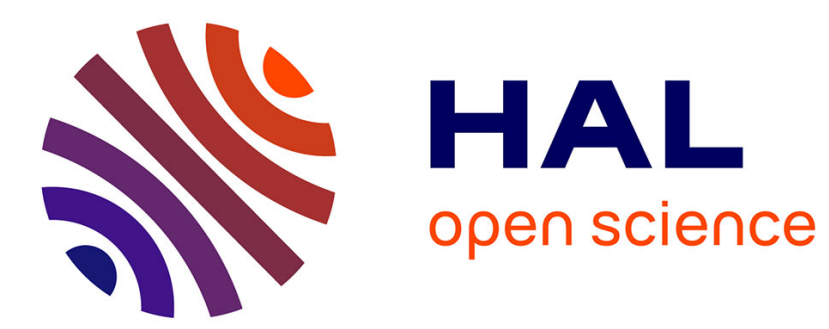

\title{
DEFECT CONFIGURATION AND ENERGY COMPUTATION IN SIMPLE METALS
}

\author{
J. Grilhé, P. Beauchamp
}

\section{To cite this version:}

J. Grilhé, P. Beauchamp. DEFECT CONFIGURATION AND ENERGY COMPUTATION IN SIMPLE METALS. Journal de Physique Colloques, 1974, 35 (C7), pp.C7-91-C7-96. 10.1051/jphyscol:1974708 . jpa-00215864

\section{HAL Id: jpa-00215864 https://hal.science/jpa-00215864}

Submitted on 1 Jan 1974

HAL is a multi-disciplinary open access archive for the deposit and dissemination of scientific research documents, whether they are published or not. The documents may come from teaching and research institutions in France or abroad, or from public or private research centers.
L'archive ouverte pluridisciplinaire HAL, est destinée au dépôt et à la diffusion de documents scientifiques de niveau recherche, publiés ou non, émanant des établissements d'enseignement et de recherche français ou étrangers, des laboratoires publics ou privés. 


\title{
DEFECT CONFIGURATION AND ENERGY COMPUTATION IN SIMPLE METALS
}

\author{
J. GRILHE and P. BEAUCHAMP \\ Laboratoire de Métallurgie Physique \\ 40, avenue du Recteur-Pineau, 86022 Poitiers, France
}

Résumé. - L'application de la méthode de Hartree et de la théorie des pseudo-potentiels permet d'écrire l'énergie des métaux normaux sous la forme :

$$
E=\sum_{i} E_{i}+\sum_{i, j} W\left(\left|\mathbf{r}_{i}-\mathbf{r}_{j}\right|\right)
$$

$r_{i}$ et $r$ désignant les positions de deux atomes.

A volume constant, $E_{\imath}$ est constant et les variations de l'énergie du cristal dépendent seulement de la contribution des interactions de paires.

Ce résultat est utilisé pour le calcul des énergies de fautes d'empilement et des configurations de cœur des dislocations dans les métaux normaux.

Abstract. - By using the Hartree method and the results of pseudo-potentials theory, the energy of simple metals can be put as follows :

$$
E=\sum_{i} E_{i}+\sum_{i, j} W\left(\left|\mathbf{r}_{i}-\mathbf{r}_{j}\right|\right)
$$

$r_{i}$ and $r_{j}$ characterizing the positions of two atoms.

At constant volume, $E_{i}$ is constant and variations of the crystal energy depend only on the pair interaction contribution.

This result has been used to compute stacking fault energies and dislocations core configurations in simple metals.

Introduction. - The dissociation of dislocations and their core configuration play important parts in the study of mechanical properties of crystals.

When stacking fault energies are small they can be determined experimentally. In the other cases, no direct techniques are available, and the computer simulation only can give some idea of these core configurations.

Many calculations were carried out in metals using pair potentials. Bragg and Williams, for ordered alloys, were among the first authors who used such potentials. The study of point defects created by radiation damage has had also a great importance in the development of such techniques.

The validity of results obtained by that method can be discussed ; the use of pair interaction is not generally justified and the potentials used (Morse potentials or polynomial potentials with parameters adjusted to some physical properties) is not realistic. Our purpose is first to review how in simple metals and for defects which do not change the total crystal volume the use of pair potentials has been justified.

In the second part we shall see how the potentials can be used for stacking fault energy calculations and for the determination of screw dislocation core configuration.
1. Pair potentials. - The first attempts to express the energy of a metal in terms of sum of interactions between pairs of ions were based on Hartree's model for metals.

1.1 HARTRE'S MODEL. - At $0 \mathrm{~K}$ we may consider a simple metal as a set of fixed pointlike ions embedded in the conduction electron gas. The total energy is then the sum of the electrostatic interaction between all the particles and of the kinetic energy of the electrons.

The interaction energy between ions can be calculated in a classical way, and is written as a sum of pair interactions.

$$
W_{k}=\sum_{j \neq k} \frac{Z^{2} e^{2}}{\left|\mathbf{R}_{j}-\mathbf{R}_{k}\right|}
$$

$\mathbf{R}_{k}$ defines the position of the $k$ ion.

To get the other part of the crystal energy, we have to solve the Schrödinger equation for the conduction electrons.

In Hartree's method one writes the Schrödinger equation for a single electron :

$$
-\frac{1}{2} \Delta \varphi_{k}(r)+\left(V_{\mathrm{i}}+V_{\mathrm{e}}\right) \varphi_{k}=E_{k} \varphi_{k}(r)
$$

in atomic units $(h=e=m)$. 
$V_{\mathrm{i}}$ is the potential created by the ions.

The interaction between the considered $k$ electron and the other conduction electrons is accounted by $V_{\mathrm{e}}$.

$V_{\mathrm{e}}$ is related to the conduction electron charge density $\rho$ through Poisson's equation.

$$
\Delta V_{\mathrm{e}}=-4 \pi \rho
$$

and $\rho$ can be expressed in terms of the single-electron wave functions $\varphi_{k}$

$$
\rho=\sum_{k \in \omega} \varphi_{k}^{*}(r) \varphi_{k}(r) .
$$

The sum is over the wave functions with a wave number inside the Fermi sphere $\omega$. The self-consistent resolution of eq. (1) and (2) gives the electron wavefunction and energies and the potential $V_{\mathrm{e}}$.

In the case of simple metals we can use a nearly free electrons approximation and calculate the energies $E_{k}$ using a perturbation method up to second order.

The sum $\sum_{k} E_{k}$ gives the electron kinetic energy, the electron-ions interaction and the electron-electron interaction counted twice. The latter can be calculated independently since we know the electron wave function and the potential $V_{\mathrm{e}}$.

$$
E_{\text {ee }}=\frac{1}{2} \int_{v} \rho V_{\mathrm{e}} \mathrm{d} v .
$$

(The integration is on the crystal total volume.)

The total energy of the crystal is :

$$
E=E_{\text {ion-ion }}+\sum_{k \in \omega} E_{k}-\frac{1}{2} \int_{v} \rho V_{\mathrm{e}} \mathrm{d} v .
$$

A calculation of these terms shows that it is possible to write the energy as

$$
E=E_{0}+\sum_{i} E_{i}\left(\mathbf{R}_{i}\right)+\frac{1}{2} \sum_{i \neq j} W\left(\left|\mathbf{R}_{i}-\mathbf{R}_{j}\right|\right) .
$$

Each of the three quantities depends on the crystal volume. At constant volume $E_{0}$ is constant, $E_{i}\left(R_{i}\right)$ depends only on the nature of the $i$ ion, and $W_{i j}\left(R_{i j}\right)$ is the effective interaction between the $i$ and $j$ atoms, function of their separation $R_{i j}$. Only the latter term is affected by a modification of the crystal structure at constant volume.

Thus, it is justified, if one is interested in a structure change (without volume change) to write the energy change as a sum of pair interactions. This result is due to the fact that the perturbation has not been carried on further than second order. A third order expansion would lead to interactions between three ions.

The pair interaction $W(r)$ is :

$$
W(r)=\frac{Z^{2} e^{2}}{2 \pi^{2}} \int \frac{\mathrm{e}^{i q r}}{q^{2} \varepsilon(q)} \mathrm{d}^{3} q
$$

$\varepsilon(q)$ is the dielectric constant of the free-electron gas.

$$
\varepsilon(q)=1+\frac{2 k_{\mathrm{F}}}{\pi q^{2}} \cdot g\left(\frac{q}{2 k_{\mathrm{F}}}\right)
$$

with

$$
g(x)=1+\frac{1}{2 x}\left(1-x^{2}\right) \log \left|\frac{1+x}{1-x}\right|
$$

where $k_{\mathrm{F}}$ is the Fermi vector.

For distances greater than the second or third nearest neighbour distance, the potential $W(r)$ has an oscillatory asymptotic form

$$
W(r) \simeq \frac{\pi}{k_{\mathrm{F}}\left(1+2 \pi k_{\mathrm{F}}\right)^{2}} \cdot Z^{2} \frac{\cos \left(2 k_{\mathrm{F}} r\right)}{r^{3}} .
$$

These oscillations are due to the screening of the bare ion potential by the conduction electrons (Friedel oscillations). They are the manifestation in the real space of a Khön singularity in the dielectric function $\varepsilon(q)$ for $q=2 k_{\mathrm{F}}$ in the reciprocal space.

For short distances a numerical calculation of the integral

$$
\int \frac{\mathrm{e}^{\prime q r}}{q^{2} \varepsilon(q)} \mathrm{d}^{3} q
$$

is necessary to obtain $W(r)$.

Thus, Hartree's model justifies the use of pair potentials for simple metals provided the volume is kept constant.

The main characteristics of these potentials are to be oscillatory and long-range.

1.2 Pseudo-Potentials. - The use of Hartree's method presents some strong limitations :

Near the ions, the potential is very strong, and it is difficult to justify the use of a perturbation method. In this region the conduction electron wave-function varies very quickly and its development in planewave requires a large number of terms.

These difficulties can be eliminated by the use of a pseudo-potential method. The metal can be divided into two types of regions, bounded by spheres centred on the atomic sites and with a radius of the order of the ionic radius (Fig. 1). Inside a sphere the conduction

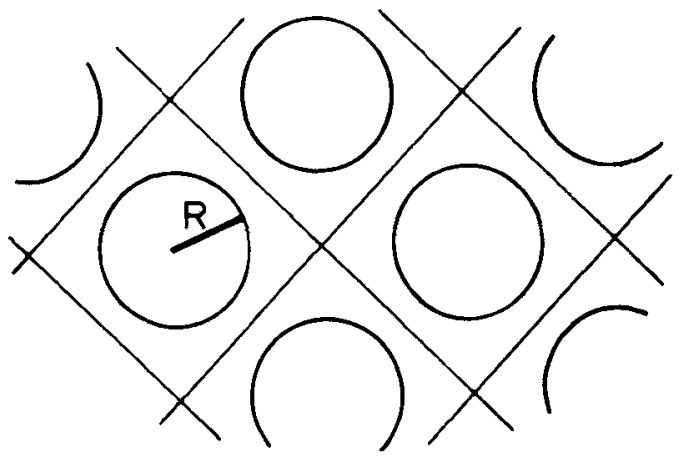

FIG. 1. - The division of the metal into two parts by spheres centered on the ions.

electrons are mainly submitted to the potential created by the ion situated in this sphere. Outside the spheres the potential is weak and comes from all the ions in the metal. 
A structure modification with constant volume will not affect the wave functions inside the ions, thus it is sufficient to know the electron cloud wave function outside to deduce the physical properties of the metal.

It is then possible to replace the actual potential inside the spheres by any potential if it gives the right wave functions outside the spheres.

There are many potentials satisfying this condition and several ways for their determination have been suggested. One of the earliest method is the orthogonalized plane waves method (OPW).

The initial purpose of this method is not to have a smaller potential, but to have the conduction electron wave functions orthogonal to the core electron wave functions $\Psi_{c}$. The $\Psi_{k}$ functions are written as :

$$
\left|\psi_{k}\right\rangle=|k\rangle+\sum_{c} a_{c k}\left|\psi_{c}\right\rangle \text {. }
$$

The sum is over the bounded states. The calculation leads to a system of equations formally similar to the system obtained in Hartree's model, the only difference being that the potential $V=V_{i}+V_{e}$ is replaced by

$$
\left.V^{\mathrm{OPW}}=V+\sum_{c}\left(E_{\imath}-E_{c}\right) ; \Psi_{c}><\Psi_{c}\right\} .
$$

This pseudo-potential is a non-local pseudo-potential. This means that $V^{\mathrm{OPW}}$ is not a simple multiplicative operator such as $V(r)$. It can be shown that if one replaces, in the Schrödinger equation, $V$ by $V^{\mathrm{OPW}}$ one gets pseudo-wave functions which differ from the actual wave functions inside the ion only (Fig. 2). The

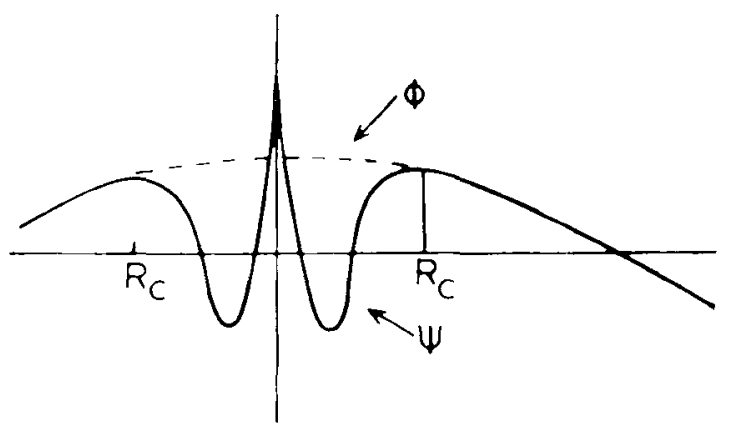

FIG. 2. - Schematic representation of the wave function $\Psi$ and the pseudo-wave function $\Phi$ of a conduction electron.

eiggen values $E_{k}$ remain unchanged. More generally it can be shown that these two properties are true for any potential which can be written as

$$
V+\sum_{c} f_{c}(r)<\Psi_{c} \mid
$$

Many potentials of this form have been used.

An other type of approach is to introduce a cut-off radius $R_{m}$ and to take for $r>R_{m}, V=-Z / r$ and to determine for $r<R_{m}$ a potential which gives the actual wave function and its first derivative for $r=R_{m}$ (Fig. 2). This can be done theoretically using, for instance, a phase shift method. Another method is to assume a simple shape for the potential, $V=$ Cte for instance and to fit this constant and the cut-off radius $R_{m}$ with some physical parameters, either calculated by an other technique, or measured. For instance, the potential can be fitted to the calculated charge density created by an isolated ion in a neutralized electron gas [1].

Among the measured parameters we can mention the energy of the valence electrons in the isolated atom or ion, the determination of the Fermi surfaces, elastic constants, phonon spectra, liquid metals resistivity, etc... It is necessary to note here that all physical properties are not sensitive to the same parameters in the interatomic potential, so that a potential fitted to a particular property does not necessarily give good results for any other property. Several authors have reviewed and discussed these problems of the choice and determination of a pseudo-potential (W. A. Harrison [2], M. L. Cohen and V. Heine [3], I. M. Torrens [4]).

1.3 EXCHANGE AND CORRELATION CORRECTIONS. When using single electron wave-functions one neglects the exchange interactions and the use of an average potential $V_{e}$ is an approximation which does not take into account the correlation between electrons.

The approximations seem to be justified as long as the mean volume per atom remains constant.

The main result of the correlation and exchange correction is to change the dielectric function of the electron gas [5].

Reliable potentials are now available for alkali metals. They lead to good results for many of the physical properties.

The typical shape of pair potentials is shown (Fig. 3) which is a potential calculated for Lithium by R. Pick [6].

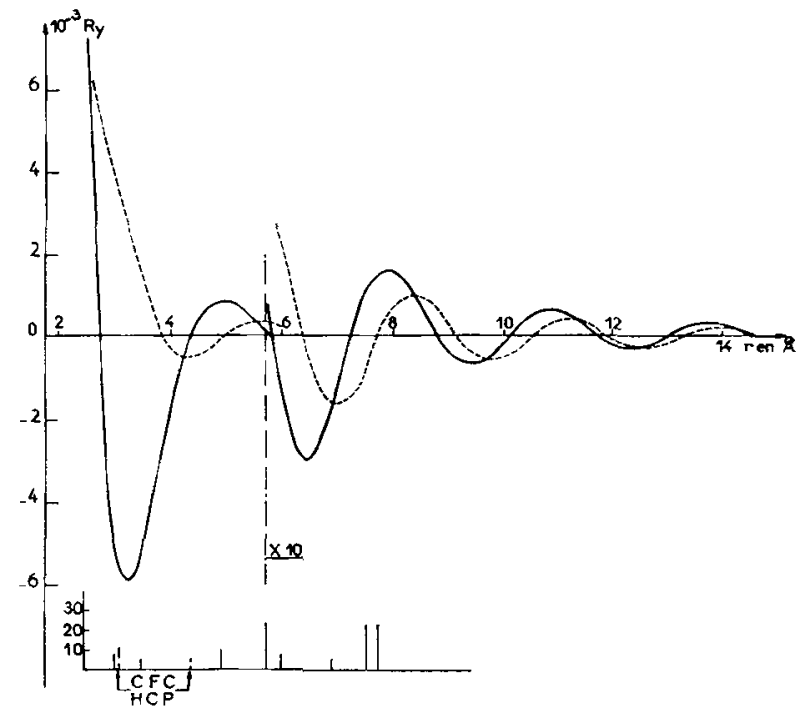

FIG. 3. - A pair potential for Lithium (R. Pick [6]). Hartree's model : - .... Pseudo potential : -

2. Application to extended structure defects. 2.1 STACKING FAULT ENERGIES IN COMPACT STRUCTURES. - Compact structures are formed by stacking 


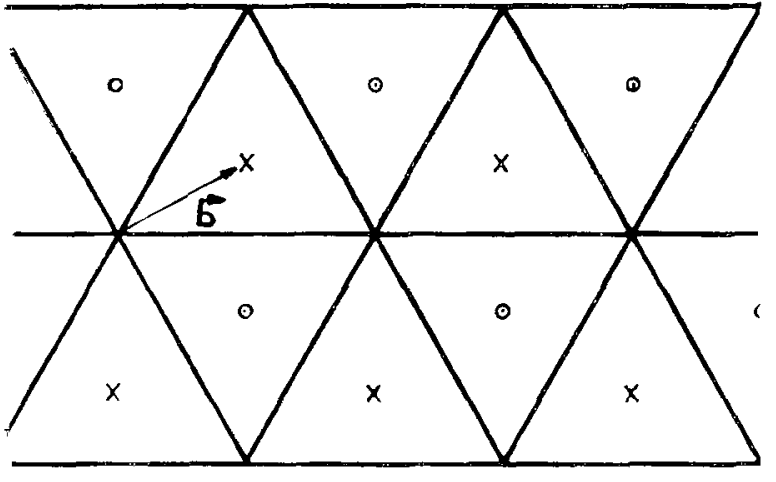

FIG. 4. - Definition of the A, B, C planes in a compact structure. - $\mathbf{A} ; \times \mathbf{B} ; \odot \mathbf{C}$.

of planes of three types A, B and C (Fig. 4). The stacking sequence of these three types of planes defines the structure or the fault in the structure (Table I).

\section{TABLE I}

$\begin{array}{ll}\text { Perfect lattice } & \text { A B C A B C } \\ \text { Intrinsic fault } & \text { A B C B C A } \\ \text { Extrinsic fault } & \text { A B C B A B } \\ \text { Spinel twin } & \text { A B C B A C }\end{array}$

a) F. C. C. structure.

$\begin{array}{ll}\text { Perfect lattice } & \text { B A B A B A B } \\ 1 \Delta \text { fault } & \text { B A B C B C B } \\ \text { Intrinsic } 2 \Delta \text { fault } & \text { B A B C A C A } \\ \text { Extrinsic } 3 \Delta \text { fault } & \text { B A B C A B A }\end{array}$

b) Hexagonal structure.

In each case, the defect can be obtained by pure shear of the crystal along the $\mathbf{b}$ type vectors (Fig. 5) contained in a dense plane. The energy of such a defect in the

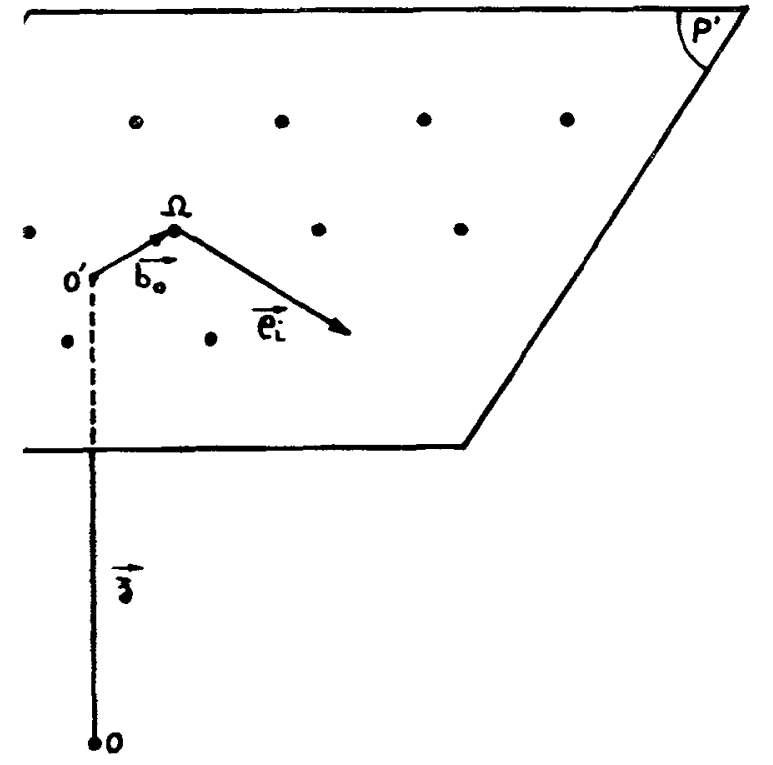

FIG. 5. - Calculation of the interaction energy between atomic planes. difference between the faulted crystal energy and the perfect crystal energy.

$$
W=\sum_{i>j} W\left(R_{i j}^{\prime}\right)-\sum_{i>j} W\left(R_{i j}\right)
$$

$R_{i j}^{\prime}$ and $R_{i j}$ are the distances between atoms in the faulted crystal and in the perfect crystal.

The interactions being long range, the calculation in real space is long; we shall present a method due to A. Blandin, J. Friedel and G. Saada [7] which avoids this difficulty.

The interatomic distances inside a plane do not change, so the interactions between atoms in this plane are not modified; thus it is not necessary to calculate these interactions. The defect energy can be written as sum of the interactions between the atoms in a P plane and the atoms in a $\mathrm{P}^{\prime}$ plane parallel to $\mathrm{P}$.

Using the Fourier transform of the pair interaction and the summation properties in the reciprocal lattice of the $P$ plane one can obtain the interaction energy between $\mathrm{P}$ and $\mathrm{P}^{\prime}, \chi\left(z, \mathbf{b}_{0}\right)$. In a translation of $\mathrm{P}^{\prime}$ by a vector $\mathbf{b}$ the change in the $\mathbf{P}-\mathbf{P}^{\prime}$ interaction energy is (per surfaces unit) :

$$
\Phi\left(z, \mathbf{b}_{0}, \mathbf{b}\right)=\frac{1}{\Omega} \chi\left(\mathbf{z}, \mathbf{b}_{0}, \mathbf{b}\right)-\chi\left(\mathbf{z}, \mathbf{b}_{0}\right) .
$$

The asymptotic form of this function of $z$ is gouverned by the singularity in the dielectric constant; for $k=2 k_{\mathrm{F}}$ one gets two different asymptotic forms: one for $|\lambda|<2 k_{\mathrm{F}}$, the other one for $|\lambda|>2 k_{\mathrm{F}}$ ( $\lambda$ is a vector of the $\mathrm{P}^{\prime}$ plane reciprocal lattice). In terms of average number of electrons per atom $Z$ there is a critical $Z_{\mathrm{c}}=1.14$; one of the asymptotic forms is valid for $Z<Z_{\mathrm{c}}$, the other one $Z>Z_{\mathrm{c}}$.

$Z<Z_{\mathrm{c}}$ (alkali metals) :

$$
\Phi\left(z, \mathbf{b}_{0}, \mathbf{b}\right)=A\left(\mathbf{b}_{0}, \mathbf{b}\right) \frac{\mathrm{e}^{-2 k_{\lambda} z}}{\left(2 k_{\mathrm{F}} z\right)^{2}}
$$

with $2 k_{\lambda}=\sqrt{\lambda^{2}-4 k_{\mathrm{F}}^{2}}$.

$Z>Z_{\text {c }}$ (it is the case for di and tri-valent metals) :

$$
\Phi\left(\mathbf{z}, \mathbf{b}, \mathbf{b}_{0}\right) \simeq-A\left(\mathbf{b}, \mathbf{b}_{0}\right) \frac{\sin \left(2 k_{\lambda} z\right)}{\left(2 k_{\mathrm{F}} z\right)^{2}}
$$

where $2 k_{\lambda}=\sqrt{4 k_{\mathrm{F}}^{2}-\lambda^{2}}$.

The fault energy reduces then to a summation of the $\Phi\left(z, \mathbf{b}_{0}, \mathbf{b}\right)$ over the different values of $z$, distance between planes.

For alkali metals, the interaction decreases exponentially; for $Z>Z_{\mathrm{c}}$ the interaction is much longer range : it decreases as $1 / z^{2}$. We must note that for short distances the asymptotic form is not valid and we have to calculate $\Phi$ numerically.

For alkali and divalent metals and for aluminium, the results are of the same order of magnitude as the experimental results, often unaccurate.

For instance, for alkali metals one finds a negligeable stacking fault energy in FCC structure and a positive but small energy for HCP structure, which is in agree- 
ment with the stability of this phase at low temperature for $\mathrm{Li}$ and $\mathrm{Na}$, and with the large number of observed stacking faults. In the case of aluminium the calculated values vary between 150 and $250 \mathrm{erg} / \mathrm{cm}^{2}$ for intrinsic stacking faults. The experimental values are around $200 \mathrm{ergs} / \mathrm{cm}^{2}$.

In the case of noble metals, the pseudo-potentials are not known well enough to give satisfactory results.

Among the numerous stacking fault energy calculations made using pair potentials from the pseudopotential theory, we can extract $V$. Vitek [8] who does the calculation in real space and allows a relaxation of the planes near the fault, and C. H. Hodges [9] who does the calculation in reciprocal space, calculating the structure factor of a faulted lattice.

\subsection{CORE CONFIGURATIONS OF SCREW DISLOCATIONS} IN BCC STRUCTURES. - This fundamental problem for the comprehension of the plastic behaviour of b. c. c. metals has been studied by the use of pseudopotentials, in the cases of lithium [11-12] and sodium [13-14].

To calculate the core configuration, one divides the crystal into two parts by a cylinder of radius $R_{\mathrm{c}}$ centred on the dislocation. For $r>R_{\mathrm{c}}$, the atoms are fixed in the position given by the elasticity. For $r<R_{\mathrm{c}}$, the atoms are relaxed so that the crystal energy is minimised. In the same way, it was done for the interactions between planes, it is possible to calculate the interaction between atomic rows $W(\rho, z), \rho$ is the distance between the two rows and $z$ the relative displacement parallel to the rows (Fig. 6).

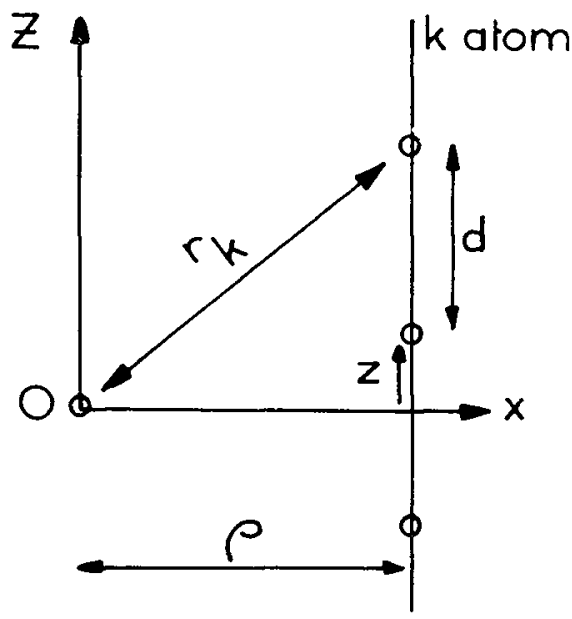

Fro. 6. - Calculation of the interaction energy between atomic rows.

$W(\rho, z)$ can be written as a Fourier expansion over the vectors $\lambda_{n}$ of the reciprocal lattice of the atomic row $\left(\lambda_{n}=n \frac{2 \pi}{d}\right)$

$$
W(\rho, z)=\sum_{n=0}^{\infty} F_{n}\left(\rho, \lambda_{n}\right) \cos \left(\lambda_{n} z\right)
$$

The expression of $F_{n}\left(\rho, \lambda_{n}\right)$ depends on the relative values of $\lambda_{n}$ and $2 k_{\mathrm{F}}$.

$$
\begin{aligned}
& \lambda_{n}<2 k_{\mathrm{F}} \\
& F_{n}\left(\rho, \lambda_{n}\right)=A \frac{\sin \left(\sqrt{\left(4 k_{\mathrm{F}}^{2}-\lambda^{2}\right)} \cdot \rho+\frac{3 \pi}{4}\right)}{\rho^{5 / 2}} \\
& \lambda_{n}>2 k_{\mathrm{F}} \\
& F_{n}\left(\rho, \lambda_{n}\right)=B \frac{\left.\exp \left(-\sqrt{\left(\lambda^{2}-4 k_{\mathrm{F}}^{2}\right.}\right) \rho\right)}{\rho^{5 / 2}} .
\end{aligned}
$$

The form of the quantities $F_{0}(\rho, \lambda)$ and $F_{1}(\rho, \lambda)$ is shown of figure 7 for Pick's potential for Lithium. The exponential decrease of the higher order coefficient makes $W(\rho, z)$ converge towards an asymptotic form. This asymptotic form depends on the value of $2 k_{\mathrm{F}}$ with respect to $\lambda=2 \pi / d$, so that there is a critical average number of conduction electrons per atom $Z_{\mathrm{c}}$ $\left(Z_{\mathrm{c}}=0.81\right.$ for $\mathrm{a}<111>$ row in a b. c. c. structure $)$.

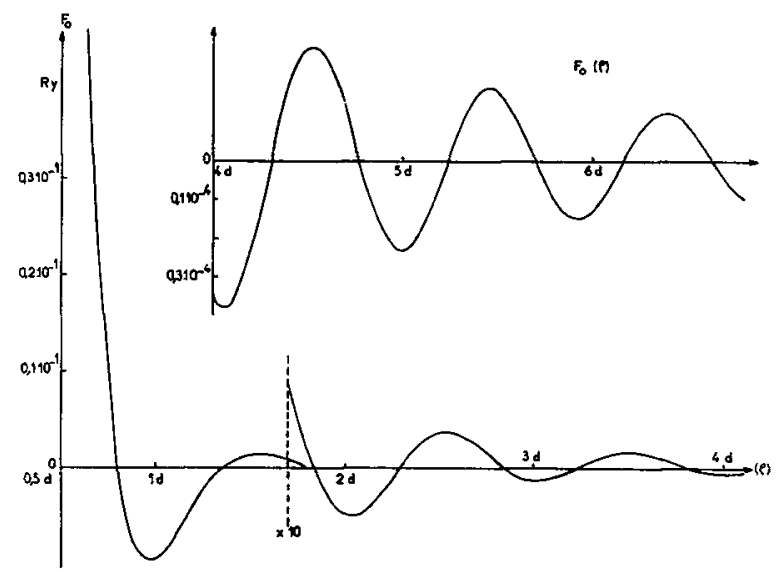

(a)

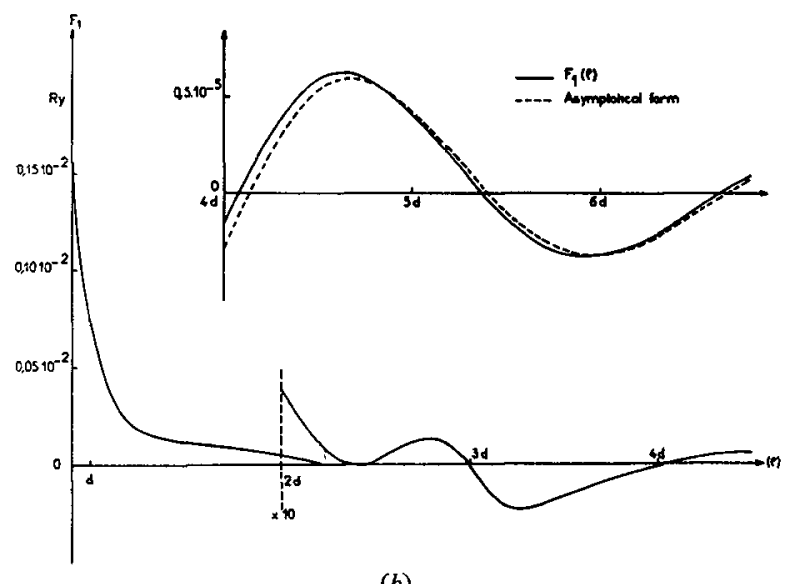

(b)

FrG. 7. - Functions $F_{0}(\rho)$ et $F_{1}(\rho, \lambda)$ in the interaction between atomic rows.

For $Z>Z_{\mathrm{c}}$ the asymptotic form writes

$W(\rho, z)=A \frac{\sin \left(k_{\lambda} \cdot \rho+\frac{3 \pi}{4}\right)}{\rho^{5 / 2}} \cos \lambda z+A_{0} \frac{\sin \left(k \cdot \rho+\frac{3 \pi}{4}\right)}{\rho^{5 / 2}}$ with $k_{\lambda}=4 k_{\mathrm{F}}^{2}-\lambda^{2}$. 
The core configurations of screw dislocations with or without stress being the subject of an other communication, we shall not discuss the results found for Liand $\mathrm{Na}$.

3. Conclusion. - In simple metals the pseudopotential theory justifies the use of pair-potential for the study of defects, under the condition that there is no volume change. The potentials are oscillatory and long range.

However, except for alkali metals, the potentials are yet uncertain. They give generally the right order of magnitude for the stacking fault energy calculations, but there is an uncertitude in the results they give for the defect configuration which require the determination of a minimum in the crystal energy. Considering the amount of research going on in this field, one can expect very reliable potentials to be available within some years.

Several authors are now interested in the influence of exchange and correlation effects; this should lead to the possibility of calculation of defects with volume changes.

\section{References}

[1] Rasolt, M. and TAYLOR, R., J. Phys. F 3 (1973) 1678

[2] Harrison, W. A., Pseudo-potentials in the theory of metals (Benjamin) 1966.

[3] Cohen, M. L. and Herne, Y., Solid. State Phys. (1970).

[4] Torrens, I. M., Interatomic potentials (Academic press) 1972.

[5] Geldart, D. L. W. and TAYlor, R., Solid State Commun. 9 (1971) 7.

[6] PICK, R., J. Physique 28 (1967) 539.

[7] Blandin, A., Friedel, J. et SAAdA, G., J. Physique Colloq. 27 (1966) C 3-128.

[8] VITEK, V., Phil. Mag. 18 (1968) 773
[9] Hodges, C. H., Phil. Mag. 15 (1967) 371.

[10] Grilhe, J. et RABIER, J., 2nd Int. Conf. on the strength of metals and alloys, Pacific Grove, Calif. 2 (1970) 431.

[11] Rabier, J. et Grilhe, J., J. Phys. Chem. Solids 34 (1973) 1031.

[12] Beauchamp, P., Rabier, J., Grilhe, J., J. Physique 34 (1973) 923.

[13] Basinski, Z. S., Ruesbery, M. S., TAYlor, R., Phil. Mag. 21 (1970) 1201.

[14] Basinski, Z. S., Duesbery, M. S. and TAYLoR, R., Interatomic potentials and simulation of lattice defects, P. C. Gehlen, J. R. Beeler and R. J. Jaffee, ed. (Plenum Press, New York) 1972, p. 537. 